\title{
Nucleosynhetic Pt isotope anomalies and the Hf-W chronology of iron meteorites
}

\author{
FRIDOLIN SPITZER ${ }^{1}$, CHRISTOPH BURKHARDT ${ }^{1}$, \\ FRANCIS NIMMO $^{2}$ AND THORSTEN KLEINE ${ }^{1}$ \\ ${ }^{1}$ University of Münster, Institut für Planetologie \\ ${ }^{2}$ University of California Santa Cruz \\ Presenting Author: fridolin.spitzer@uni-muenster.de
}

The accretion of planetesimals is the first step towards forming planets, and so understanding when and where planetesimals formed is crucial for constraining early Solar System dynamics. The formation of planetesimals can be dated using the short-lived Hf-W system by determining an age of core formation, which in turn can be used to estimate the time of parent body accretion via thermal modelling. For instance, the Hf-W chronology of 'magmatic' iron meteorites indicates that they derive from bodies formed within first $\sim 1 \mathrm{Ma}$ of the Solar System, and that core formation in the parent bodies of non-carbonaceous (NC) iron meteorites (which formed in the inner disk) occurred earlier than in those of carbonaceous (CC) iron meteorites (which formed in the outer disk) [1]. Determining Hf-W ages for iron meteorites also requires the quantification of superimposed cosmic rayinduced $\mathrm{W}$ isotope shifts. This is commonly achieved using Pt isotopes as the neutron dosimeter, and by assuming that all $\mathrm{Pt}$ isotope variations are due to cosmic ray-effects [1]. Here we show, however, that bulk meteorites have small nucleosynthetic $\mathrm{Pt}$ isotope anomalies, and that once these are taken into account, $\mathrm{Hf}-\mathrm{W}$ model ages for both $\mathrm{NC}$ and $\mathrm{CC}$ irons become $\sim 1 \mathrm{Ma}$ younger. Nevertheless, we find no resolvable nucleosynthetic $\mathrm{Pt}$ isotope difference between $\mathrm{NC}$ and $\mathrm{CC}$ meteorites, and so even after this correction, the Hf-W ages for $\mathrm{CC}$ irons are still $\sim 1-2$ $\mathrm{Ma}$ younger than those of $\mathrm{NC}$ irons. To assess whether this difference reflects different accretion times, we calculated the thermal evolution of planetesimals heated internally by ${ }^{26} \mathrm{Al}-$ decay. As the $\mathrm{CC}$ iron meteorite parent bodies most likely formed at or beyond the snowline, we also quantified the effect of water ice on the time of core formation. We find that the distinct core formation times of $\mathrm{NC}$ and $\mathrm{CC}$ iron meteorite parent bodies can be accounted for by different water ice fractions in these bodies, implying that early and rapid planetesimal formation occurred about contemporaneously in the inner and outer disk.

[1] Kruijer T. S. et al. (2017) PNAS, 114, 6712-6716. 